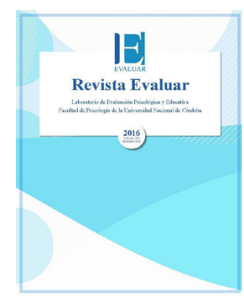

\title{
Estudio psicométrico del instrumento CARLOS (CRAFFT) en estudiantes universitarios mexicanos
}

\section{Psychometric study of the CARLOS (CRAFFT) instrument in Mexican university students}

\author{
Gloria Velia Reyna-Barajas * ${ }^{1}$, Luis Fernando Copertari-Isaacson ${ }^{1}$, \\ Fabiola González-Betanzos ${ }^{2}$, Ferran Padrós-Blàzquez ${ }^{2}$
}

1 - Universidad Autónoma de Zacatecas, Zacatecas, México.

2 - Universidad Michoacana de San Nicolás de Hidalgo, Morelia, México.

\section{Resumen}

En México existen instrumentos con adecuadas propiedades psicométricas que evalúan el consumo de alcohol, así como diversos aspectos asociados a su consumo y percepción de riesgo (véase Calleja, 2011). Sin embargo, hasta ahora no se ha identificado una escala creada o validada en el país que mida al mismo tiempo el consumo de alcohol y otras sustancias psicoactivas de forma breve y precisa. El objetivo de este trabajo fue examinar las propiedades psicométricas del instrumento CARLOS (CRAFFT) de Knight, Sherrit, Shrier, Harris y Chang (2002) en una muestra de 220 estudiantes universitarios mexicanos. Se aplicaron los instrumentos CARLOS (CRAFFT) y AUDIT a estudiantes universitarios. Se optó por la técnica del cuestionario auto-administrado. Se aplicaron los instrumentos dentro del salón de clases. Se asignó 1 a la respuesta afirmativa y 0 a la respuesta negativa. La edad promedio de los participantes fue de 20 años (rango 18 a 25 años). El instrumento CARLOS (CRAFFT) mostró niveles moderadamente altos de consistencia interna (Alfa de Cronbach $=$ .73). El punto de corte de 2 ofrece la mejor combinación de sensibilidad (.86) y especificidad (.73) con valor predictivo positivo (.52) y valor predictivo negativo (.93). El área bajo la curva (ROC) fue de .79. La correlación $r$ de Pearson con el instrumento AUDIT fue de $.67(\mathrm{p}<.01)$. El instrumento CARLOS (CRAFFT) es suficientemente válido y confiable para la tamización del consumo de sustancias psicoactivas en estudiantes universitarios mexicanos

Palabras clave: CARLOS (CRAFFT), sustancias psicoactivas, cribado, estudiantes universitarios mexicanos
Introducción

Método

Resultados

Discusión

Referencias

\begin{abstract}
In Mexico there are instruments with adequate psychometric properties evaluating alcohol consumption, as well as several aspects of its use and risk perception (refer to Calleja, 2011). However, so far a scale measuring both alcohol consumption and other psychoactive substances in a brief and precise way has not been validated in Mexico. This study aimed to examine the psychometric properties of the CARLOS (CRAFFT) instrument by Knight, Sherrit, Shrier, Harris and Chang (2002) in a sample of 220 Mexican university students. The instruments CARLOS (CRAFFT) and AUDIT were applied to Mexican university students. The self-administered test technique was used and the instruments were applied inside the classroom. 1 was assigned to the affirmative answer and 0 to the negative answer. The average age of the participants was 20 years (ranging from 18 to 25 years). The CARLOS (CRAFFT) instrument showed moderately high internal consistency levels (Cronbach's alpha $=.73$ ). The cutoff point of 2 offers the best combination of sensibility (.86) and specificity (.73) with a positive predictive value (.52) and a negative predictive value (.93). The area under the ROC curve was .79 and Pearson's $r$ correlation between the CARLOS (CRAFFT) and the AUDIT instrument was .67 $(\mathrm{p}<.01)$. The CARLOS (CRAFFT) instrument is sufficiently valid and reliable for testing psychoactive substances consumption in Mexican university students.
\end{abstract}

Keywords: CARLOS (CRAFFT), psychoactive substances, screening, Mexican university students

\footnotetext{
*Correspondencia a: Gloria Velia Reyna-Barajas. Dirección: Calle de los Faroles 110, Fracc. Colonial Zacatecas, Zacatecas, Zac. 98068, México. Teléfono: +52 (492) 156-4240. gloriavelia@yahoo.com.mx

Cómo citar este artículo:Reyna-Barajas, G. V., Copertari-Isaacson, L. F., González-Betanzos, F., \& Padrós-Blàzquez, F. (2016). Estudio psicométrico del instrumento CARLOS (CRAFFT) en estudiantes universitarios mexicanos. Evaluar, 16, 10-19.Recuperado de https://revistas.unc.edu.ar/index.php/revaluar
} 


\section{Introducción}

A nivel internacional el uso de drogas psicoactivas es un problema de salud pública (Kračmarová, Klusoňová, Petrelli, \& Grappasonni, 2011). Según el Informe Mundial sobre Consumo de Drogas (2013) presentado por la Oficina de las Naciones Unidas contra la Droga y el Delito (UNODC, por sus siglas en inglés United Nations Office on Drugs and Crime), el cannabis es la sustancia ilícita típicamente más consumida a nivel internacional, registrando un leve incremento en la prevalencia de los consumidores. A este le sigue el consumo de estimulantes del tipo de las anfetaminas presente en todo el mundo y cuyo consumo está creciendo en todas las regiones. El consumo de la cocaína está disminuyendo en Europa y Estados Unidos, pero se está incrementando su uso en América del Sur y las economías emergentes de Asia.

Según la Encuesta Nacional de Adicciones (ENA, 2011), en México el abuso de sustancias psicoactivas constituye un problema actual de salud pública, que afecta a niños y adolescentes de ambos géneros y de distintos niveles socioeconómicos de todo el país. Aún así, México es uno de los países con menor consumo de drogas a nivel internacional, aunque datos relativamente recientes indican un aumento en el consumo de drogas en ambos géneros, en el que destacan los varones (Villatoro et al., 2012).

En México existen instrumentos con adecuadas propiedades psicométricas que evalúan el consumo de alcohol y otras sustancias psicoactivas, así como diversos aspectos asociados a su consumo y percepción de riesgo (veáse Calleja, 2011). Sin embargo, hasta ahora no se ha identificado una escala creada o validada en el país que detecte y evalúe de forma breve y precisa el consumo de este tipo de sustancias.

La escala CARLOS (CRAFFT) desarro- llada por Knight et al. (2002) es un instrumento de aplicación muy breve que ha manifestado aceptables propiedades psicométricas. En el estudio original de Knight et al. (2002), el punto de corte óptimo de la Escala CRAFFT resultó ser igual o superior a 2, con una sensibilidad de .76 y una especificidad de .94. El alfa de Cronbach resultó ser de .68 .

En el estudio de Cummins et al. (2003), llevado a cabo en Alaska, para un punto de corte igual o superior a 2, la sensibilidad resultó ser de .95 y la especificidad de .86; el alfa de Cronbach fue de .81 .

En la validación francesa Bernard et al. (2005) reportaron que para un punto de corte igual o superior a 4 , la sensibilidad resultó ser igual a .72 y la especificidad de .68 .

En un estudio hecho en Singapur, con una muestra multiétnica de Malasia, India y China, con un punto de corte igual o superior a 2, se encontró una sensibilidad de .64 y una especificidad de .84, la escala mostró un alfa de Cronbach de .73 (Subramaniam, Cheok, Verma, Wong, \& Chong, 2010).

En Noruega, Skogen, Bøe, Knudsen y Hysing (2013), reportaron que para un punto de corte igual o superior a 2 , la sensibilidad fue de .41 , la especificidad de $.90 \mathrm{y}$ el alfa de Cronbach fue de .67 .

En la validación para Colombia, Cote-Menendez, Uribe-Isaza y Prieto-Suárez (2013), reportaron una sensibilidad de $\mathbf{9 5}$, una especificidad de .83 , el alfa de Cronbach resultó de .89 .

En la adaptación transcultural y validación hecha en Argentina se reportó que con un puntaje mayor o igual a 2, el instrumento CRAFFT tuvo una sensibilidad del 59\%, una especificidad del 88\% y una consistencia interna de .68.

El objetivo del presente estudio fue examinar las propiedades psicométricas del ins- 
trumento CARLOS (CRAFFT) en una muestra de estudiantes de la Universidad Autónoma de Zacatecas. Por lo tanto, se calculó la consistencia interna del instrumento, se valoró la sensibilidad y especificidad de la prueba, se determinaron los valores predictivos y se examinó su estructura factorial.

\section{Método}

Participantes

La muestra de investigación está conformada por 220 estudiantes de diferentes Unidades Académicas de la Universidad Autónoma de Zacatecas.

Para determinar el tamaño de la muestra, en un primer momento, se siguió la recomendación de Cortina (1993) y Norman y Streiner (1996) la cual indica que: "cuando el número de participantes calculado para una escala compuesta por menos de diez ítems es inferior a $100 \mathrm{su}-$ jetos, se recomienda que la muestra se forme con diez participantes por cada ítem [...]" (como se cita en Campos-Arias \& Oviedo, 2008, p. 836). Esta recomendación puede ser empleada cuando se realizan estudios de consistencia interna y análisis factorial exploratorio (Campos-Arias \& Oviedo, 2008).

Siguiendo la anterior indicación, la muestra de este estudio estaría compuesta por 60 participantes. Sin embargo, se consideró conveniente incrementar el tamaño de la muestra para reducir el margen de error de las estimaciones (Argibay, 2009). En consecuencia, se utilizó una muestra por conveniencia de 220 participantes.

De los 220 participantes, el 58\% (127) son mujeres y el $42 \%$ (93) son varones. El rango de edad oscila entre 18 y 25 años (media de 20.1, desviación estándar de 1.6, moda de 19). Proceden de las siguientes facultades: Psicología,
35\% (77); Odontología, 17\% (37); Ingeniería en Computación, 14\% (31); Contaduría y Administración, 14\% (31); Ingeniería Civil, 11\% (25) y Economía, 9\% (19).

\section{Instrumentos}

\section{Las Preguntas CARLOS/CRAFFT (Carro,} Amigos, Relajarse, Líos, Olvidado, Solo / Car, Relax, Alone, Forget, Friends, Trouble.) De Knight et al. (2002); CARLOS o CRAFFT son acrónimos de las primeras letras de las palabras clave del test de seis preguntas. Se utilizó la versión oficial en español, realizada por los autores del instrumento, que consiste en un instrumento de cribado que permite identificar adolescentes en riesgo de abuso de sustancias. Está compuesto por seis ítems (sección B: B1, B2, B3, B4, B5 y B6), aunque hay otros tres adicionados al principio a efectos de realizar una rápida tamización (sección A: A1, A2, A3). El formato de respuesta es dicotómico (Sí o $\mathrm{No}$ ). Si se responde que no a los primeros tres ítems de tamizado (A1, A2, A3) se debe responder solamente el primero de los seis ítems de diagnóstico (B1). Si se responde que sí a cualquiera de los tres ítems de tamizado se deben responder los seis ítems de diagnóstico (B1 a B6). Los seis ítems de diagnóstico del instrumento cuestionan la presencia o ausencia de consumo de alcohol u otras drogas y pregunta por los problemas que se relacionan con el consumo de estas. En caso de responder de forma negativa (no) se le asigna un puntaje de cero, mientras que a una respuesta afirmativa (sí) se le asigna un puntaje de uno. Para evaluar el instrumento se suman los puntajes de los seis ítems de diagnóstico (del B1 al B6). Una puntuación mínima de 0 o 1 respuestas afirmativas se considera como punto de corte (indicativa de ausencia de riesgo de consumo problemático de sustan- 
cias). Puntajes iguales o mayores a 2 sugieren la presencia de consumo abusivo (Clark, Gordon, Ettaro, Owens, \& Moss, 2010).

Las preguntas CARLOS (CRAFFT) han sido valoradas psicométricamente en diferentes estudios durante más de una década (Pérez-Gómez \& Scopetta-Díaz-Granados, 2011) y se ha demostrado su validez a través de distintos estudios (Cote-Menendez, et al., 2013).

Para hacer uso de las Preguntas CARLOS (CRAFFT), se solicitó el correspondiente permiso a los autores del instrumento, que fue concedido a través de Jesse Boggis, Ph.D., Research Assitant II, Center for Adolescent Substance Abuse Research (CeASAR), Boston Children's Hospital, Harvard Medical School, MA, USA.

\section{AUDIT (por sus siglas en inglés, Alcohol Use} Disorders Identification Test). De Saunders, Aasland, Babor, De la Fuente y Grant (1993), adaptación mexicana de Rubio (1998), es un instrumento conformado por 10 ítems. Las primeras ocho preguntas tienen cinco opciones de respuestas, que se puntúan de 0 a 4 y las dos últimas preguntas tienen tres opciones de respuesta que se puntúan 0, 2 o 4. El rango de respuestas es de 0 a 40 . Las primeras tres preguntas exploran la cantidad y frecuencia del consumo de bebidas alcohólicas. Una alta puntuación en estos ítems sugiere que la persona está bebiendo alcohol de manera abusiva. Una alta puntuación en las respuestas de las preguntas 4 a 6 muestra que existe dependencia de consumo de alcohol. Si existen altas puntuaciones en las preguntas 7 a 10 se lo considera como un consumo dañino o perjudicial. El punto de corte para considerar riesgo, consumo perjudicial o incluso dependencia de alcohol es de 8 puntos o más en el total de los puntos obtenidos en los 10 ítems (Babor, Higgins-Biddle, Saunders, \& Monteiro, 2001).

\section{Procedimiento}

El estudio constó de tres fases: en la primera tres psicólogos expertos revisaron la traducción oficial al español realizada por los autores del instrumento CARLOS (CRAFFT) y consideraron que es adecuada.

En la segunda fase se hizo un estudio piloto a 30 estudiantes de Psicología de las Preguntas CARLOS (CRAFFT) con el objetivo de detectar posibles dificultades en la comprensión y respuesta de las preguntas del instrumento y para medir el tiempo que se ocupa en responderlo. Los participantes expresaron entender ampliamente todas las preguntas, no manifestaron dudas y lo contestaron en un lapso de tiempo de uno a tres minutos.

En la tercera fase se realizó el estudio a escala donde participaron 220 estudiantes y se aplicaron los instrumentos CARLOS (CRAFFT) y AUDIT. En general, se siguió el siguiente procedimiento:

Se optó por la técnica del cuestionario auto-administrado, por lo que se procedió a aplicar los instrumentos de forma colectiva dentro del salón de clases. Se explicó a los estudiantes los objetivos del estudio. Se especificó el carácter confidencial de los datos aportados. Se les informó de la importancia de responder de forma verídica a las distintas cuestiones planteadas. Quienes participaron lo hicieron de forma voluntaria y firmaron el consentimiento informado. No hubo límite de tiempo para responder a cada uno de los instrumentos aplicados; sin embargo, este osciló entre 5 y 10 minutos. Por orden de aplicación, en el cuadernillo de los instrumentos primero aparece el AUDIT y después el CARLOS (CRAFFT). 
Análisis de datos

Primero se estimó la consistencia interna del instrumento CARLOS (CRAFFT) con el alfa de Cronbach. Enseguida, y en consonancia con el estudio previo de Knight et al. (2002), se calcularon la sensibilidad, la especificidad, los valores predictivos del instrumento y las áreas bajo la curva ROC, para determinar el punto de corte óptimo para predecir cualquier uso problemático de alcohol o drogas.

Para verificar la validez concurrente se calculó la correlación del instrumento CARLOS (CRAFFT) con el AUDIT. Por último y considerando los resultados del análisis factorial exploratorio (AFE) del instrumento CRAFFT realizado por Subramaniam et al. (2010) y cuyos resultados indican que el instrumento es unifactorial, se procedió a realizar un análisis factorial confirmatorio (AFC) del instrumento mediante Modelos de Ecuaciones Estructurales (MEE) considerando que todos los reactivos se agrupan en un solo factor.

\section{Resultados}

Del total de los participantes, el 33\% informó no haber consumido sustancias. Los porcentajes de los participantes que respondieron de manera afirmativa ( $s i$ ) a cada ítem del CARLOS (CRAFFT) y los porcentajes que presentaron puntuaciones de 0 a 6 se presentan en las Tablas 1 y 2 , respectivamente.

\section{Tabla 1}

Porcentaje de participantes que respondieron afirmativamente (si) a cada ítem de Las Preguntas CARLOS (CRAFFT).

\begin{tabular}{lc}
\hline \multicolumn{1}{c}{ Ítem } & $\begin{array}{c}\text { Respuesta sí a cada ítem de Las } \\
\text { Preguntas CARLOS }\end{array}$ \\
\hline Carro & $59 \%$ \\
Amigos & $28 \%$ \\
Relajarse & $20 \%$ \\
Líos & $16 \%$ \\
Olvidado & $22 \%$ \\
Solo & $19 \%$ \\
\hline
\end{tabular}

Tabla 2

Porcentaje de participantes que tuvieron puntuaciones de 0 a 6.

\begin{tabular}{cc}
\hline $\begin{array}{c}\text { Puntuación CARLOS } \\
\text { (CRAFFT) }\end{array}$ & Porcentaje \\
\hline 0 & $33 \%$ \\
1 & $25 \%$ \\
2 & $13 \%$ \\
3 & $11 \%$ \\
4 & $11 \%$ \\
5 & $6 \%$ \\
6 & $1 \%$ \\
\hline
\end{tabular}

Análisis de confiabilidad y estudio de la bondad de los reactivos

Se estimó la consistencia interna del instrumento CARLOS (CRAFFT) y se obtuvo un alfa de Cronbach de .73 que indica, teniendo en cuenta el reducido número de reactivos, que la consistencia interna de la escala es aceptable (Aiken, 2003). La media y la desviación estándar del puntaje total fue de 1.63 y 1.65 respectivamente. La Tabla 3 muestra un resumen de los resultados obtenidos por reactivo. La puntuación media para cada ítem osciló entre .15 (ítem B4) y .59 (ítem B1).

L estándar para cada ítem estuvieron entre .36 y .49 . El rango de correlaciones de cada uno de los ítems con la puntuación total corregida no presentó valores negativos o iguales a 0 , estuvieron por encima de .41 , excepto el reactivo B6 que resultó de .37. También se observó que al 
Los valores de las desviaciones estándar para cada ítem estuvieron entre .36 y .49. El rango de correlaciones de cada uno de los ítems con la puntuación total corregida no presentó valores negativos o iguales a 0 , estuvieron por encima de .41, excepto el reactivo B6 que resultó de 37 . También se observó que al eliminar cualquiera de los ítems, el valor del alfa de Cronbach de la escala total disminuía considerablemente (véase Tabla 3). punto de corte de 2. Al mismo punto de corte, el valor predictivo positivo resultó de .52 y el valor predictivo negativo de .94 . El área bajo la curva (ROC) fue de .79 (.50 - .79).

El punto de corte es aquel valor límite a partir del cual se considera que se tiene un caso de abuso de sustancias psicoactivas. Se busca obtener el punto de corte óptimo para que el balance entre sensibilidad y especificidad sea

Tabla 3

Estadísticos básicos y confiabilidad de los resultados obtenidos del CARLOS (CRAFFT).

\begin{tabular}{lcccc}
\hline Reactivo & Media & $\begin{array}{c}\text { Desviación } \\
\text { estándar }\end{array}$ & $\begin{array}{c}\text { Correlación elemento-total } \\
\text { corregida }\end{array}$ & $\begin{array}{c}\text { Alfa de Cronbach si se } \\
\text { elimina } \\
\text { el elemento }\end{array}$ \\
\hline $\mathrm{C}-\mathrm{B} 1$ & 0.59 & 0.49 & 0.41 & 0.71 \\
$\mathrm{~A}-\mathrm{B} 2$ & 0.28 & 0.45 & 0.50 & 0.68 \\
$\mathrm{R}-\mathrm{B} 3$ & 0.20 & 0.39 & 0.50 & 0.68 \\
$\mathrm{~L}-\mathrm{B} 4$ & 0.15 & 0.36 & 0.51 & 0.68 \\
$\mathrm{O}-\mathrm{B} 5$ & 0.22 & 0.41 & 0.53 & 0.67 \\
$\mathrm{~S}-\mathrm{B} 6$ & 0.19 & 0.39 & 0.37 & 0.72 \\
\hline
\end{tabular}

Sensibilidad, Especificidad, Valor Predictivo Positivo (VPP), Valor Predictivo Negativo (VPN) y Curvas ROC para diferentes puntos de corte del instrumento CARLOS (CRAFFT)

Se hizo un estudio de la variación a los diferentes puntos de corte del CARLOS (CRAFFT) al considerar diferentes totales del instrumento como casos positivos y compararlos con los casos positivos del AUDIT. Dichos resultados se muestran en la Tabla 4.

La sensibilidad de una prueba es la probabilidad de clasificar correctamente a un individuo que presenta la enfermedad mientras que la especificidad es la probabilidad de que una persona sana tenga un resultado negativo en la prueba (Cuevas-Renaud \& Alejo-Martínez, 2010). Así se encuentra que la sensibilidad del CARLOS (CRAFFT) comparado con el AUDIT fue de .86 , y su especificidad de .73 con un el mejor posible, es decir, que la prueba mida de manera más precisa el abuso de sustancias psicoactivas ( $\sin$ errores ya sea por omisión o diligencia).

La curva ROC resultante de graficar 1-Especificidad en el eje horizontal y Sensibilidad en el eje vertical para los diferentes puntos de corte (Hanley \& McNeil, 1982), se ilustra en la Figura 1.

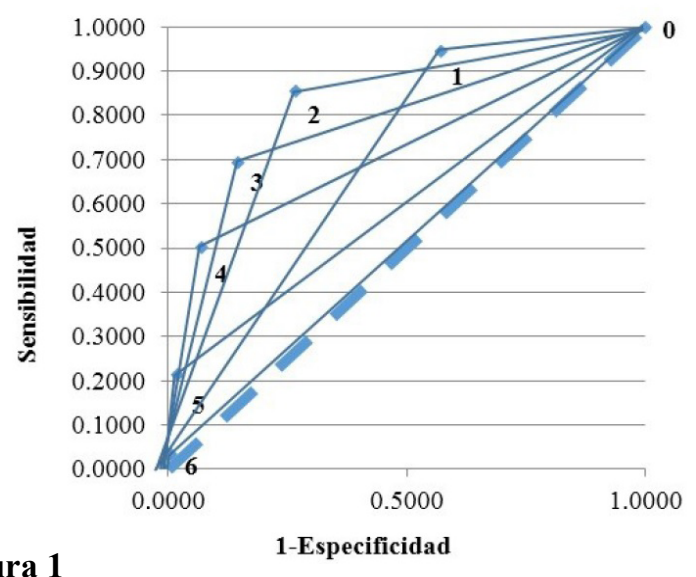

Figura 1

Curvas ROC para los diferentes puntos de corte CARLOS (CRAFFT). 
Tabla 4

Análisis de diferentes puntos de corte CARLOS (CRAFFT).

\begin{tabular}{llllllll}
\hline Corte & Sensibilidad & $\begin{array}{l}\text { Especifici- } \\
\text { dad }\end{array}$ & $\begin{array}{l}\text { Valor Predictivo } \\
\text { Positivo }\end{array}$ & $\begin{array}{l}\text { Valor Predictivo } \\
\text { Negativo }\end{array}$ & $\begin{array}{l}\text { 1-Especificidad bajo } \\
\text { Kappa curva } \\
\text { ROC }\end{array}$ \\
\hline 0 & 1.0000 & 0.0000 & 0.2545 & 1.0000 & 1.0000 & & -0.5000 \\
1 & 0.9464 & 0.4268 & 0.3605 & 0.9589 & 0.5732 & 0.2530 & 0.6866 \\
2 & 0.8571 & 0.7317 & 0.5217 & 0.9375 & 0.2683 & 0.4860 & 0.7944 \\
3 & 0.6964 & 0.8537 & 0.6190 & 0.8917 & 0.1463 & 0.5280 & 0.7750 \\
4 & 0.5000 & 0.9329 & 0.7179 & 0.8453 & 0.0671 & 0.4810 & 0.7165 \\
5 & 0.2143 & 0.9817 & 0.8000 & 0.7854 & 0.0183 & 0.2580 & 0.5980 \\
6 & 0.0357 & 0.9939 & 0.6667 & 0.7512 & 0.0061 & 0.0430 & 0.5148 \\
\hline
\end{tabular}

Nota. Se observa que el punto de corte de 2 es el que ofrece la mejor combinación de sensibilidad y especificidad, así como la mayor área bajo la curva ROC, a pesar de que la mayor Kappa sea para un punto de corte de 3.

Validez concurrente de las escalas CARLOS (CRAFFT) y AUDIT

Para la validez concurrente se hizo una correlación para los 220 individuos de la suma de los seis ítems del CARLOS (CRAFFT; B1, B2, B3, B4, B5 y B6) respecto a la suma de los 10 ítems del AUDIT. La correlación $\mathrm{r}$ de Pearson para este caso resultó de $.67(p<.01)$.

\section{Análisis factorial confirmatorio (AFC)}

El análisis factorial exploratorio llevado a cabo por Subramaniam et al. (2010) mostró que todos los ítems de la escala CRAFFT se cargaron bajo un mismo factor con un peso factorial que va de .60 a .93. Más aún, el modelo unifactorial cumplió con todos los criterios de un buen modelo. Por consiguiente en este estudio se espera encontrar un solo factor. Para hacer el AFC se utilizó un Modelo de Ecuaciones Estructurales o SEM (por sus siglas en inglés, Structural Equation Model) aplicado al instrumento CARLOS (CRAFFT). Debido a que el comportamiento del instrumento no sigue una distribución normal, se utilizó el método de mínimos cuadrados generalizados (generalized least squares) en lugar del método de máxima verosimilitud (maximum likelihood) debido a que el primero no requiere un comportamiento normal de las variables. El resultado se muestra en la Figura 2.

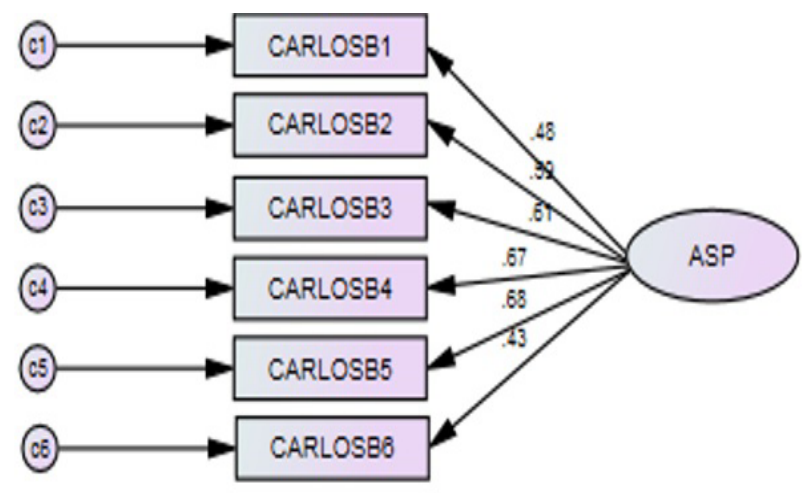

Figura 2

MEE del AFC del CARLOS (CRAFFT).

Nota. ASP significa Abuso de Sustancias Psicoactivas. 


\section{Discusión}

El primer objetivo del presente trabajo fue evaluar la consistencia interna del instrumento CARLOS (CRAFFT). Los resultados indican una consistencia interna aceptable (alfa de Cronbach de .73). El resultado obtenido es igual al presentado en el estudio de Subramaniam et al. (2010) y comparable a los valores obtenidos en otros estudios. Por ejemplo en el estudio original de Knight et al. (2002) se reportó un alfa de Cronbach de .68; Skogen et al. (2013) señalaron un alfa de Cronbach de .67; Bertini et al. (2014), indicaron un alfa de Cronbach de .64; Cummins et al. (2003) reportaron .81. En la revisión realizada por Dhalla, Zumbo y Poole (2011) se encontraron valores que van de .65 a .86 , mientras que en el estudio de Kandemir et al. (2015) se obtuvo un alfa de Cronbach de .90. Por lo tanto, al igual que en los estudios citados anteriormente, se concluye que el instrumento es apropiado para la detección de uso y abuso de alcohol y otras substancias.

El segundo objetivo fue evaluar la sensibilidad y la especificidad del instrumento. En este estudio se encontró que hay una mayor sensibilidad y especificidad y mejor área bajo la curva (ROC) con un punto de corte de 2 , al igual que lo expuesto en otros estudios como los de Bertini et al. (2014), Cote-Menéndez et al. (2013), Cook, Chung, Kelly y Clark (2004), Cummins et al. (2003), Kandermir et al. (2015), Knight et al. (2002), Mitchell et al. (2014) y Tossman, Kasten, Lang y Strüber (2009). Nótese en este estudio que con un punto de corte de 2 , la sensibilidad del CARLOS (CRAFFT) (.86) fue mayor a la reportada por Knight et al. (2002; .76) y la especificidad (.73) fue menor a la reportada por los autores citados (.94). Sin embargo, los resultados encontrados en el presente estudio tienen mayor similitud a los reportados en Alemania por Tossmann et al. (2009). Ellos indicaron que para un punto de corte igual o superior a dos, obtuvieron una sensibilidad y especificidad de .89 y .66 , respectivamente. Por otro lado, en una revisión sistemática de las propiedades psicométricas del CRAFFT que incluye 11 estudios de validez y 6 estudios de fiabilidad que se llevaron a cabo en diferentes tipos de poblaciones, los resultados indican que es un buen instrumento para detectar abuso de sustancias, incluyendo el consumo problemático, abuso y dependencias. En los puntos de corte óptimos, se obtuvo una sensibilidad con rangos de .61 a 1.00, mientras que la especificidad variaba de .33 a .97 (Dhalla et al., 2011). Los resultados del presente estudio indican que el instrumento CARLOS (CRAFFT) tiene una adecuada sensibilidad y especificidad. Por lo tanto, se puede concluir que el instrumento CARLOS (CRAFFT) como prueba diagnóstica es conveniente, se puede confiar en el diagnóstico clínico obtenido a través de la aplicación del instrumento.

El tercer objetivo fue determinar los valores predictivos. En el presente estudio se encontró un VPP $=.52$, es decir, con una probabilidad de acierto del $52 \%$ se predice el consumo de sustancias psicoactivas. El VPN $=.94$ indica que con un $94 \%$ de certidumbre el abuso de sustancias psicoactivas no está presente. Es necesario resaltar que para ambos valores predictivos se consideró un punto de corte de 2 . Los resultados de los valores predictivos son similares a los reportados por Cook et al. (2004), quienes indican que con un punto de corte igual o mayor a 2 , se obtuvo un VPP $=.41$ y un VPN $=.92$.

Por último, al igual que en el estudio de Subramaniam et al. (2010), se observa en este trabajo la estructura unifactorial del instrumento CARLOS (CRAFFT). Nótese que no existen 
covarianzas significativas entre los errores de las seis variables exógenas (B1 a B6) en el MEE. El punto de corte de 2 es el que ofrece mejores índices sobre la validez discriminante de la escala.

Los resultados que se han obtenido en otros estudios de las propiedades psicométricas del instrumento CRAFFT son muy similares a los obtenidos en la validación hecha al contrastar el instrumento CARLOS (CRAFFT) y la encuesta AUDIT en población mexicana. Los resultados del presente estudio muestran una fiabilidad, sensibilidad y especificidad suficientemente altas. Por lo tanto, se concluye que los resultados muestran que el instrumento CARLOS (CRAFFT) es válido para la tamización de consumo de alcohol y otras sustancias en jóvenes mexicanos.

La principal limitación del estudio se refiere a que la muestra utilizada sólo está compuesta por estudiantes universitarios. Además, debe tenerse presente que sólo se ha aplicado en la ciudad de Zacatecas. Sería recomendable realizar estudios con muestras más representativas de otros estados de la República Mexicana y que tengan diferentes características sociodemográficas.

Otra importante limitación es la ausencia de una medición adecuada para estudiar la validez concurrente (la escala AUDIT evalúa sólo el consumo abusivo de alcohol). En futuros estudios sería conveniente utilizar otros Gold Standards (por ejemplo, una entrevista estructurada). Por otro lado, sería interesante estudiar la fiabilidad test-retest del instrumento, así como la sensibilidad al cambio después de intervenciones.

\section{Conclusión}

Los datos obtenidos en el presente estudio sugieren que el instrumento CARLOS (CRAFFT) es suficientemente válido y confiable para la tamización del consumo de sustancias psicoactivas en estudiantes universitarios mexicanos.

\section{Referencias}

Aiken, L. R. (2003). Test psicológicos y evaluación (10 ed.). México: Pearson Educación.

Argibay, J. C. (2009). Muestra en investigación cuantitativa. Subjetividad y Procesos Cognitivos, 13(1), 13-29. Recuperado de http://dspace.uces.edu.ar:8180/xmlui/handle/123456789/719

Babor, T., Higgins-Biddle, J., Saunders, J., \& Monteiro, M. (2001). AUDIT Cuestionario de identificación de los trastornos debidos al consumo de alcohol: Pautas para su utilización en atención primaria. Suiza: Organización Mundial de la Salud, Departamento de Salud Mental y Dependencias de Sustancias.

Bernard, M., Bolognini, M., Plancherel, B., Chinet, L., Laget, J., Stephan, P., \& Halfon, O. (2005). French validity of two substance-use screening tests among adolescents: A comparison of the CRAFFT and DEPADO. Journal of Substance Use, 10(6), 385-395. doi: 10.1080/14659890412331333050

Bertini, M. C., Busaniche, J., Baquero, F., Eymann, A., Krauss, M., Paz, M., \& Catsicaris, C. (2014). Adaptación transcultural y validación del test CRAFFT como prueba de pesquisa para consumo problemático, abuso y dependencia de alcohol y otras sustancias en un grupo de adolescentes argentinos. Recuperado de http://www.sap.org.ar/docs/publicaciones/primero/2015/AO_Bertini_anticipo_12-2-15.pdf

Byrne, B. M. (2010). Structural Equation Modeling with AMOS: Basic Concepts, Applications and Programming (2 ${ }^{\mathrm{a}}$ ed.). Routledge, Taylor \& Francis Group: New York, NY.

Calleja, N. (2011). Inventario de escalas psicosociales en México 1984-2005. México: UNAM, Facultad de Psicología.

Campos-Arias, A., \& Oviedo, H. C. (2008). Propiedades psicométricas de una escala: La consistencia interna. Revista Salud Pública, 10(5), 831-839. doi: 10.1590/s012400642008000500015

Clark, D. B., Gordon, A. J., Ettaro, L. R., Owens, J. M., \& Moss, H. B. (2010). Screening and brief intervention for underage drinkers. Mayo Clinic Proceedings, 85(4), 380-391. doi: 
$10.4065 / \mathrm{mcp} .2008 .0638$

Cook, R. L., Chung, T., Kelly, T. M., \& Clark, D. B. (2005). Alcohol screening in Young persons attending a sexually transmitted disease clinic. Journal of General Internal Medicine, 20(1), 1-6. doi: 10.1111/j.1525-1497.2005.40052.x

Cote-Menendez, M., Uribe-Isaza, M. M., \& Prieto-Suárez, E. (2013). Validación para Colombia de la escala Crafft para tamización de consumo de sustancias psicoactivas en adolescentes. Revista de Salud Pública 15(2), 220-232. Recuperado de http://revistas.unal.edu.co/index.php/revsaludpublica

Cuevas-Renaud, C., \& Alejo-Martínez, A. (2010). Validez y fiabilidad de medidas de exposición y medición. México: UNAM, Recuperado de http://www.psicol.unam.mx/Investigacion2/pdf/SENSIBILIDAD \%20Y\%20ESPECIFICIDAD.pdf

Cummins, L. H., Chan, K. K., Burns, K. M., Blume, A. W., Larimer, M., \& Marlatt, G. A. (2003). Validity of the CRAFFT in American-Indian and Alaska-Native adolescents: Screening for drug and alcohol risk. Journal of Studies on Alcohol, 64(5), 727-32. doi: 10.15288/jsa.2003.64.727

Dhalla, S., Zumbo, B. D., \& Poole, G. (2011). A review of the psychometric properties of the CRAFFT instrument: 1999-2010. Current Drug Abuse Review, 4(1), 57-64. doi: $10.2174 / 1874473711104010057$

Encuesta Nacional de Adicciones (2011). Drogas ilícitas. Recuperado de http:/www.conadic.salud.gob.mx/pdfs/ ENA_2011_DROGAS_ILICITAS_.pdf

Hanley, J. A., \& McNeil, B. J. (1982). The meaning and use of the area under a receiver operating characteristic (ROC) curve. Radiology, 143(1), 29-36. doi: 10.1148/radiology.143.1.7063747

Kandemir, H., Aydemir, Ö., Ekinci, S., Selek, S., Kandemir, S. B., \& Bayazit, H. (2015). Validity and reliability of the Turkish version of CRAFFT substance abuse screening test among adolescents. Neuropsychiatric Disease and Treatment, 11, 1505-1509. doi: 10.2147/ndt.s82232

Knight, J. R., Sherritt, L., Shrier, L. A., Harris, S. K., \& Chang, G. (2002). Validity of the CRAFFT substance abuse screening test among adolescent clinic patients. Archives on Pediatric and Adolescent Medicine, 156(6), 607-614. doi: 10.1001/archpedi.156.6.607

Kračmarová, L., Klusoňová, H., Petrelli, F., \& Grappasonni, I.
(2011). Tobbaco, alcohol and ilegal substances: Experiences and attitudes among Italian university students. Revista da Associação Médica Brasileira, 57(5), 513-518. doi: 10.1016/s0104-4230(11)70105-1

Mitchell, S. G., Kelly, S. M., Gryczynski, J., Myers, C. P., O'Grady, K. E., Kirk, A. S., \& Schwartz, R. P. (2014). The CRAFFT cut-points and DSM-5 criteria for alcohol and other drugs: A re-evaluation and re-examination. Substance Abuse, 35(4), 376-380. doi: 10.1080/08897077.2014.936992

Pérez-Gómez, A., \& Scopetta-Díaz-Granados, O. (2011). El CRAFFT/CARLOS como instrumento para la identificación temprana de consumo de alcohol y otras SPA: Una adaptación al español. Revista Colombiana de Psicología, 20(2), 265-274. Recuperado de http://revistas.unal.edu.co/ index.php/psicologia

Rubio, G. (1998).Validación de la prueba para la identificación de trastornos por el uso de alcohol (AUDIT) en atención primaria. Revista Clínica Especializada, 198, 11-14.

Skogen, J. C., Bøe, T., Knudsen, A. K., \& Hysing, M. (2013). Psychometric properties and concurrent validity of the CRAFFT among Norwegian adolescents. Ung@hordaland, a population based study. Addictive Behaviors, 38(10), 2500-2505. doi: 10.1016/j.addbeh.2013.05.002

Subramaniam, M., Cheok, C., Verma, S., Wong, J., \& Chong, S. A. (2010). Validity of a brief screening instrument-CRAFFT in a multiethnic Asian population. Addictive Behaviors, 35(12), 1102-4. doi: 10.1016/j.addbeh.2010.08.004

Tossmann, P., Kasten, L., Lang, P., \& Strüber, E. (2009). Determination of the concurrent validity of the CRAFT-d - A screening instrument for problematic alcohol consumption. Zeitschrift für Kinder und Jugendpsychiatrie Psychotherapie, 37(5), 451-459. doi: 10.1024/1422-4917.37.5.451

United Nations Office on Drugs and Crime (2013). World Drug Report. Recuperado de http://www.unodc.org/unodc/secured/wdr/wdr2013/World_Drug_Report_2013.pdf

Villatoro, J., Medina-Mora, M. E., Fleiz-Bautista, C., Moreno-López, M., Oliva-Robles, N., Bustos-Garmiño, M., ... Amador-Buenabad, N. (2012). El consumo de drogas en México: Resultados de la Encuesta Nacional de Adicciones, 2011. Salud Mental, 35(6), 447-457. Recuperado de http://revistasaludmental.mx/index.php/salud_mental 